scattering points at the positions of glob centers can be derived from the $|F(\mathbf{s})|^{2}$ 's of the real crystal through dividing by $G\langle I\rangle$ in the range $2 r_{i j}<d(h k l)<r_{g g^{\prime}}$, provided that the globs in the crystal are 'spherical' and all alike. This raises the question of the existence of spherical globs in crystals.

One can say at once that the glob concept is useful only in connection with crystals with large unit cells as there must be a large number of interplanar spacings greater than $3 \AA$. The unit cells of such crystals contain a large number of atoms, ${ }^{*}$ which can be grouped into globs in many different ways. If, in interpreting the values of $\langle I\rangle$, it is assumed that the glob factors are spherical $\left(F_{g}(\mathbf{s})=F_{g}(s)\right)$, the globs themselves must be (nearly) spherical globs and correspond to only certain ways of dividing the atoms in the cell into groups. It is probable that some structures do not provide any way of assigning the atoms to groups so as to make spherical globs, but it seems likely that many complex structures can provide nearly spherical globs by some-method of subdivision. The assumption that $\langle I\rangle=N F_{g}^{2}(s)$ corresponds to selecting a method of distributing:the atoms into spherical globs, all with the same sets of interatomic distances.

When the structure of a crystal can be thought of as a set of similar globs in various positions and with different orientations, each of the structure factors is very probably near the value

$$
F(s)=\sum_{j=1}^{G} \overline{F_{g}(\mathbf{s})} \exp \left[2 \pi i s . r_{g}\right]
$$

* In orgänic crystals, the number of reflections with $d>3 \AA$ is about ten times the number of atoms in the unit cell, not counting hydrogen. obtained from formula (7) by replacing each $F_{g}(\mathbf{s})$ by its average over all orientations. This statement is true, even if the globs are quite far from being spherical, provided enough different orientations of the globs are present. In such a case:

$$
\langle I\rangle=\sum_{g=1}^{N}\left(\overline{F_{g}(\mathbf{s})}\right)^{2}=G\left(\overline{F_{g}(\mathbf{s})}\right)^{2},
$$

which is almost the same as (11), because

$$
\overline{\frac{\sin 2 \pi s r_{i j^{\prime}}}{2 \pi s r_{j j^{\prime}}}}=\frac{\sin 2 \pi s r_{j}}{2 \pi s r_{j}} \cdot \frac{\sin 2 \pi s r_{j^{\prime}}}{2 \pi s r_{j^{\prime}}},
$$

where the bar over the left hand term indicates averaging $r_{j j^{\prime}}$ over all directions of $\mathbf{r}_{j}$ and $\mathbf{r}_{j^{\prime}}$. Consequently, a satisfactory approximation to a point-glob crystal is obtained by dividing the $|F(\mathbf{s})|^{2}$ 's by $G\langle I\rangle$ in this case also, which can occur quite frequently in complicated crystals.

This work was supported by The Dean Langmuir Foundation, The Rockefeller Foundation, The Polytechnic Institute of Brooklyn and The International Business Machines Corporation; great thanks are due to all these institutions.

\section{References}

Warren, B. E. \& Gingrich, N. S. (1934). Phys. Rev. 46, 368.

Wilson, A. J. C. (1942). Nature, Lond. 150, 152.

Wilson, A. J. C. (1949). Acta Cryst. 2, 318.

Acta Cryst. (1953). 6, 736

\title{
The Structure of Diphenylene Naphthacene
}

\author{
By A. Bennett and A. W. Hanson \\ Physics Department, College of Technology, Manchester 1, England
}

(Received 2 May 1953)

\begin{abstract}
The unit cell of diphenylene naphthacene is monoclinic, with $a=11 \cdot 0 \pm 0 \cdot 05, b=5 \cdot 15 \pm 0.03$, $c=19 \cdot 6 \pm 0 \cdot 10 \AA, \beta=126^{\circ}$. The space group is $P 2_{1} / c$, and in the unit cell are two molecules, each lying on a centre of symmetry. The structure was solved by optical-transform methods, and the atomic positions were found with the aid of the $(010)$ and (100) Fourier projections.
\end{abstract}

\section{Introduction}

This compound is one of a number investigated largely by optical diffraction methods (Hanson, Taylor \& Lipson, 1952). The procedure by which the approximate structure was determined is discussed elsewhere (Hanson, Lipson \& Taylor, 1953), and will not be considered here.

\section{Unit cell and space group}

The sample supplied consisted of a number of lathlike, blue, opaque crystals; although these were extremely thin, and showed a slight tendency to bend, no difficulty was experienced in selecting single crystals suitable for $\mathrm{X}$-ray examination. 


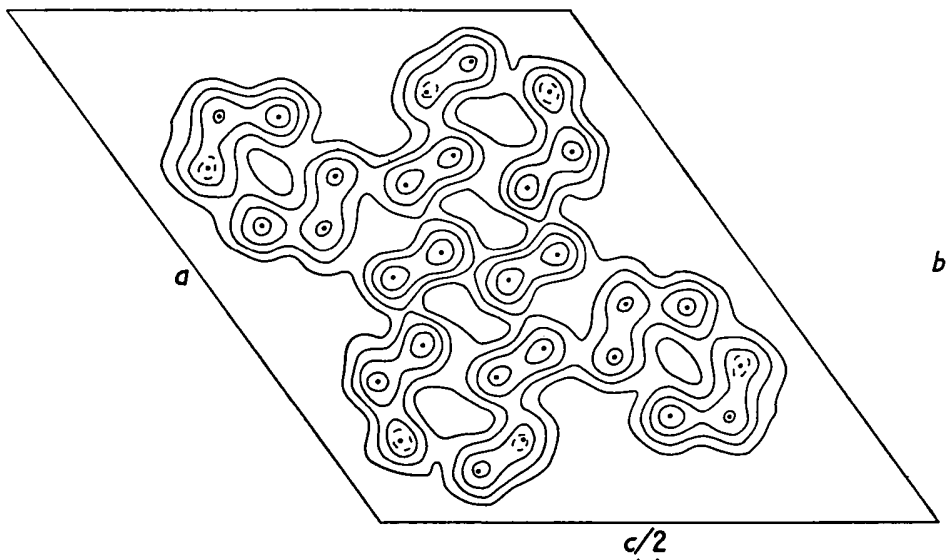

(a)

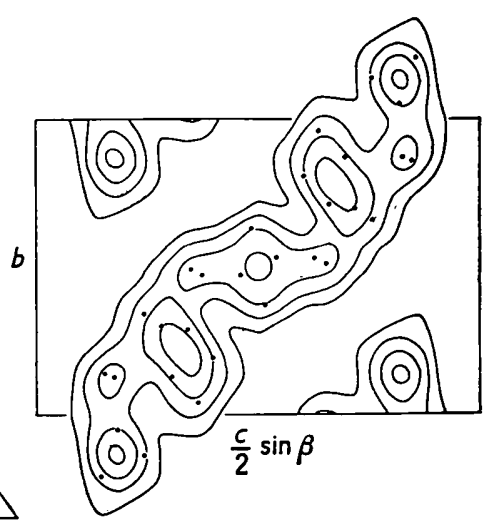

(b)

Fig. 1. (a) Electron-density map of (010) projection. Contours are drawn at intervals of 1 e. $\AA^{-2}$, the lowest being 2 e. $\AA^{-2}$. The contour at $4 \frac{1}{2} \theta . \AA^{-2}$ has been drawn (as a broken line) where necessary to indicate atomic positions. :(b) Electron-density map of (100) projection. Contours are drawn at intervals of $2 \Theta . \AA^{-2}$, the lowest being 4 e. $\AA^{-2}$.

Table 1. Atomic coordinates

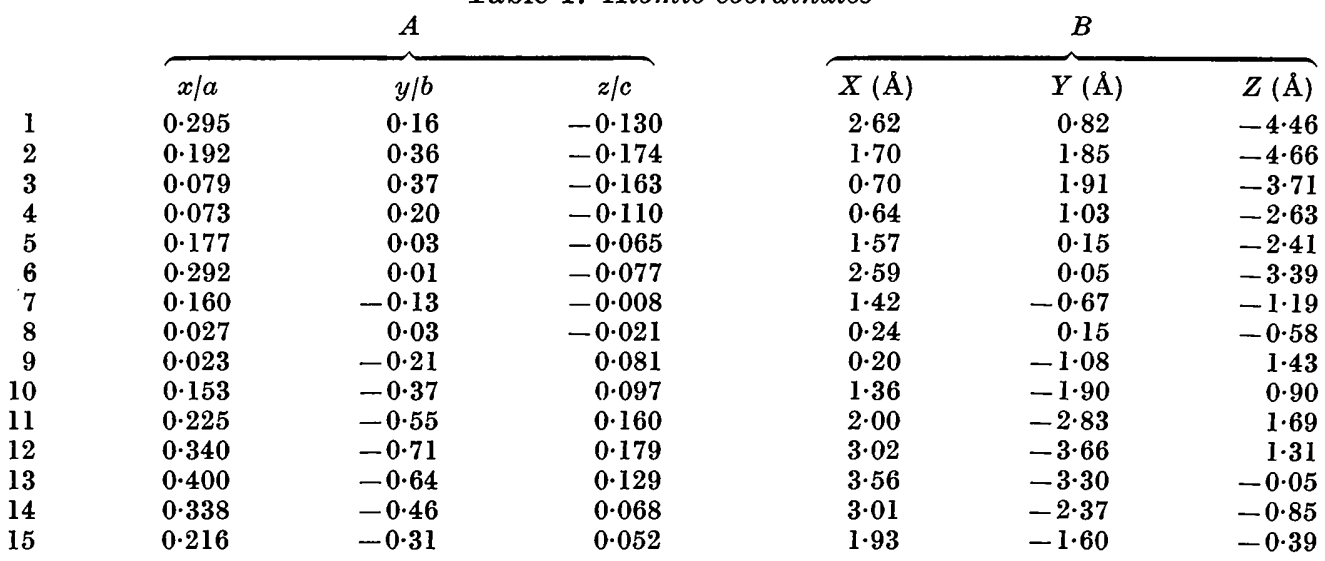

$A$ : Fractional coordinates, corresponding to crystal axes.

$B$ : Coordinates in Ångström units, referred to orthogonal axes for which $Y$ is parallel to $b, Z$ parallel to $c$.

The unit cell was shown by oscillation and Weissenberg photographs to be monoclinic, with

$$
\begin{gathered}
a=11 \cdot 0 \pm 0 \cdot 05, b=5 \cdot 15_{ \pm} 0 \cdot 03, c=19 \cdot 6 \pm 0 \cdot 10 \AA, \\
\beta=126^{\circ} .
\end{gathered}
$$

A higher degree of accuracy is not claimed because no allowance was made for film shrinkage.

The measured density of the sample was $1 \cdot 37 \pm 0 \cdot 03$ g.cm. ${ }^{-3}$, and the calculated density, corresponding to two molecules in the unit cell, was $1 \cdot 39 \pm 0.02$ g.cm. ${ }^{-3}$.

The systematic absences were $h 0 l$ for $l$ odd, and $0 k 0$ for $k$ odd. These indicated the space group $P 2_{1} / c$, with molecules lying on centres of symmetry.

\section{Determination of the structure}

The X-ray intensities for the $h 0 l$ and $0 k l$ zones were estimated by visual comparison of each Weissenberg spot with a density wedge on similar film. Very long exposures, limited ultimately by background scattering, were required for the Weissenberg photographs, and it is felt that the effect of the reflexions which were too weak to be observed might not have been negligible. Moreover, the tendency of the crystals to bend resulted in spots which differed appreciably in shape and size, some being more diffuse than others. It is feared therefore that the intensity data may not be altogether reliable.

The structure amplitudes were derived by using the usual trigonometrical factor, and, since the absorption coefficient of the compound was small, it was felt that further corrections were unnecessary.

The $(010)$ projection was readily solved by what might now be called conventional optical-transform methods. It was refined by successive sign determinations and correction syntheses; the calculation involved in determining signs was very much reduced by the use of a form of the procedure for the optical derivation of structure amplitudes (Hanson, 1953). The electron-density map of the projection is given in Fig. $1(a)$.

The (100) projection was obtained largely by stereo- 
Table 2. Observed and calculated structure amplitudes for hol zone

\begin{tabular}{|c|c|c|c|c|c|c|c|c|}
\hline$h 0 l$ & $F_{o}$ & $F_{c}$ & $h 0 l$ & $F_{o}$ & $F_{c}$ & $h 0 l$ & $F_{o}$ & $F_{c}$ \\
\hline 002 & $50 \cdot 3$ & $62 \cdot 8$ & $30 \overline{2}$ & $6 \cdot 3$ & $2 \cdot 8$ & $6,0, \overline{18}$ & $<6.4$ & $-1 \cdot 2$ \\
\hline 004 & $58 \cdot 0$ & $-60 \cdot 0$ & $30 \overline{4}$ & $5 \cdot 0$ & -8.4 & 700 & & \\
\hline 006 & $7 \cdot 8$ & $4 \cdot 8$ & $30 \overline{6}$ & $22 \cdot 4$ & $-27 \cdot 4$ & 702 & $7 \cdot 9$ & $8 \cdot 8$ \\
\hline 008 & $15 \cdot 8$ & $-15 \cdot 4$ & $30 \overline{8}$ & $24 \cdot 5$ & $-23 \cdot 8$ & 704 & $<6.4$ & 0.9 \\
\hline $0,0,10$ & $<5.2$ & $-2 \cdot 0$ & $3,0, \overline{1} \overline{0}$ & $<4.5$ & -0.7 & $70 \underline{2}$ & & $4 \cdot 4$ \\
\hline $0,0,12$ & $13 \cdot 5$ & $13 \cdot 9$ & $3,0, \overline{1} \overline{2}$ & $8 \cdot 0$ & -7.7 & $70 \overline{4}$ & $<5.5$ & $3 \cdot 9$ \\
\hline $0,0,14$ & $<6.3$ & 1.6 & $3,0, \overline{14}$ & $<5.9$ & $-3 \cdot 4$ & $70 \overline{6}$ & 12.5 & $-12 \cdot 1$ \\
\hline $0,0,16$ & $<6.4$ & $3 \cdot 8$ & $3,0, \overline{16}$ & $9 \cdot 7$ & $11 \cdot 2$ & $70 \overline{8}$ & $<5.3$ & $2 \cdot 8$ \\
\hline 100 & $72 \cdot 0$ & $67 \cdot 0$ & $3,0, \overline{1} \overline{8}$ & $<6.4$ & -1.7 & $7,0, \overline{1} \overline{0}$ & & $1 \cdot 7$ \\
\hline 102 & $28 \cdot 2$ & $29 \cdot 8$ & $3,0, \overline{2} \overline{0}$ & $13 \cdot 4$ & $-11 \cdot 3$ & $7,0, \underline{\overline{1} \mathbf{2}}$ & $<5.7$ & $-0 \cdot 8$ \\
\hline 104 & $7 \cdot 6$ & $4 \cdot 4$ & 400 & $7 \cdot 3$ & $-2 \cdot 2$ & $7,0, \overline{1 \overline{1}}$ & $11 \cdot 9$ & $14 \cdot 1$ \\
\hline 106 & $<4.2$ & $-2 \cdot 2$ & 402 & $27 \cdot 0$ & $21 \cdot 6$ & $7,0, \overline{16}$ & $<6.3$ & $-1 \cdot 7$ \\
\hline 108 & $<5.0$ & -0.4 & 404 & $6 \cdot 5$ & $6 \cdot 4$ & $7,0, \overline{18}$ & $14 \cdot 1$ & -15.9 \\
\hline $1,0,10$ & $12 \cdot 8$ & $9 \cdot 3$ & 406 & $13 \cdot 6$ & -16.9 & $7,0, \overline{2} \overline{0}$ & $<6.1$ & $-4 \cdot 5$ \\
\hline $1,0,12$ & $9 \cdot 0$ & $9 \cdot 3$ & 408 & $<6.3$ & $-1 \cdot 4$ & 800 & $8 \cdot 6$ & -8.7 \\
\hline $1,0,14$ & $<5.0$ & $-1 \cdot 0$ & $40 \overline{2}$ & $27 \cdot 4$ & $-19 \cdot 2$ & 802 & $<6.3$ & $1 \cdot 8$ \\
\hline $10 \underline{2}$ & $30 \cdot 4$ & 40.5 & $40 \overline{\underline{4}}$ & $<3.7$ & $2 \cdot 7$ & 804 & $<5.9$ & $3 \cdot 8$ \\
\hline $10 \overline{4}$ & $5 \cdot 7$ & $5 \cdot 1$ & $40 \overline{6}$ & $25 \cdot 7$ & $34 \cdot 1$ & $80 \overline{2}$ & $<6.3$ & -3.7 \\
\hline $10 \overline{6}$ & $5 \cdot 1$ & $8 \cdot 2$ & $40 \overline{8}$ & $14 \cdot 3$ & -11.9 & $80 \overline{4}$ & $<6.1$ & 0.7 \\
\hline $10 \overline{8}$ & $<4.2$ & 0.5 & $4,0, \overline{1} \overline{0}$ & $33 \cdot 6$ & $-38 \cdot 0$ & $80 \overline{\overline{6}}$ & $13 \cdot 2$ & -16.4 \\
\hline $1,0, \overline{10}$ & $8 \cdot 6$ & $4 \cdot 4$ & $4,0, \overline{1} \overline{2}$ & $<5.1$ & -0.9 & $80 \overline{8}$ & $8 \cdot 8$ & -7.3 \\
\hline $1,0, \overline{12}$ & $11 \cdot 2$ & $9 \cdot 0$ & $4,0, \overline{14}$ & $<5.8$ & $2 \cdot 2$ & $8,0, \overline{1} \overline{0} 0$ & $<5.9$ & $5 \cdot 2$ \\
\hline $1,0, \overline{14}$ & $<6.2$ & $-5 \cdot 0$ & $4,0, \overline{16}$ & $<6.3$ & $-3 \cdot 6$ & $8,0, \overline{12}$ & $<6.0$ & $2 \cdot 2$ \\
\hline $1,0, \overline{16}$ & $9 \cdot 2$ & -8.7 & $4,0, \overline{1} \overline{8}$ & $<6.4$ & $3 \cdot 2$ & $8,0, \underline{\mathbf{1 4}}$ & $<6.2$ & 3.3 \\
\hline $1,0, \overline{18}$ & $<6.3$ & -1.7 & $4,0, \overline{2} \overline{0}$ & $<5.9$ & -0.7 & $8,0,16$ & $9 \cdot 1$ & $-9 \cdot 2$ \\
\hline 200 & $52 \cdot 6$ & $-47 \cdot 0$ & 500 & $20 \cdot 5$ & $12 \cdot 7$ & $8,0, \overline{1} \overline{8}$ & $9 \cdot 7$ & $-12 \cdot 3$ \\
\hline 202 & $31 \cdot 0$ & $31 \cdot 0$ & 502 & $15 \cdot 6$ & $16 \cdot 0$ & $8,0, \overline{20}$ & $<6.1$ & -1.7 \\
\hline 204 & $7 \cdot 1$ & $-3 \cdot 8$ & 504 & $<5.7$ & $5 \cdot 1$ & 900 & $<6.2$ & $-2 \cdot 1$ \\
\hline 206 & $8 \cdot 3$ & $.4 \cdot 0$ & 506 & $<6.3$ & 0.3 & 902 & $7 \cdot 1$ & $-8 \cdot 1$ \\
\hline 208 & $26 \cdot 2$ & $23 \cdot 0$ & $50 \overline{2}$ & 6.7 & $-3 \cdot 8$ & 904 & $<4.8$ & $1 \cdot 2$ \\
\hline $2,0,10$ & $6 \cdot 3$ & $4 \cdot 4$ & $50 \overline{4}$ & $16 \cdot 3$ & $12 \cdot 2$ & $90 \overline{2}$ & $8 \cdot 0$ & $7 \cdot 2$ \\
\hline $2,0,12$ & $<6.2$ & -0.5 & $50 \overline{6}$ & $12 \cdot 1$ & $17 \cdot 3$ & $90 \overline{\underline{4}}$ & $<6.4$ & $3 \cdot 1$ \\
\hline $20 \overline{2}$ & $35 \cdot 2$ & $-41 \cdot 7$ & $50 \overline{8}$ & $6 \cdot 5$ & $1 \cdot 4$ & $90 \underline{\overline{6}}$ & $<6.2$ & -0.4 \\
\hline $20 \overline{4}$ & $28 \cdot 4$ & 35.5 & $5,0, \overline{10}$ & $6 \cdot 4$ & $-7 \cdot 0$ & $9 \underline{0 \overline{8}}$ & $17 \cdot 7$ & $-17 \cdot 2$ \\
\hline $20 \overline{\overline{6}}$ & $14 \cdot 1$ & $-14 \cdot 6$ & $5,0, \overline{12}$ & $13 \cdot 2$ & $-12 \cdot 0$ & $9,0, \underline{10}$ & $22 \cdot 4$ & $-22 \cdot 2$ \\
\hline $20 \overline{8}$ & $5 \cdot 9$ & $3 \cdot 4$ & $5,0, \overline{14}$ & $<5.7$ & $-2 \cdot 8$ & $9,0, \underline{12}$ & $<6.3$ & 0.4 \\
\hline $2,0,10$ & 18.5 & $20 \cdot 0$ & $5,0, \overline{16}$ & $<6.2$ & $4 \cdot 3$ & $9,0, \overline{14}$ & & 0 \\
\hline $2,0,12$ & $7 \cdot 7$ & -7.6 & 600 & $15 \cdot 0$ & $13 \cdot 3$ & $9,0, \overline{16}$ & $<6.4$ & -5.8 \\
\hline $2,0, \overline{14}$ & $<6.1$ & -2.5 & 602 & $<6.0$ & $0 \cdot 6$ & $9,0, \overline{18}$ & $<6.2$ & $2 \cdot 5$ \\
\hline $2,0, \overline{16}$ & $<6.4$ & $-3 \cdot 8$ & 604 & $<6.3$ & $2 \cdot 3$ & $10,0,0$ & $<5.4$ & $1 \cdot 1$ \\
\hline $2,0,18$ & $10 \cdot 8$ & $-10 \cdot 3$ & $60 \overline{2}$ & $32 \cdot 0$ & 28.5 & $10,0,2$ & $<4.3$ & -6.6 \\
\hline $2,0,20$ & $<5.2$ & $-4 \cdot 1$ & $60 \overline{4}$ & 21.7 & $22 \cdot 6$ & $10,0, \overline{2}$ & 11.9 & $7 \cdot 3$ \\
\hline 300 & 11.9 & $10 \cdot 8$ & $60 \overline{\overline{6}}$ & 10.5 & $-13 \cdot 1$ & $10,0, \overline{4}$ & $<6.3$ & -1.9 \\
\hline 302 & $<4.0$ & $-3 \cdot 2$ & $60 \overline{8}$ & $14 \cdot 6$ & $-18 \cdot 6$ & $10,0, \underline{\overline{6}}$ & $<6.4$ & $1 \cdot 2$ \\
\hline 304 & 14.9 & $-12 \cdot 8$ & $6,0, \overline{10}$ & $<5 \cdot 0$ & $8 \cdot 3$ & $10,0, \overline{8}$ & $<6.4$ & 1.2 \\
\hline 306 & $<5.3$ & -0.4 & $6,0, \overline{12}$ & $<5.4$ & -1.4 & $10,0, \overline{10}$ & $16 \cdot 8$ & -16.8 \\
\hline 308 & $16 \cdot 1$ & $7 \cdot 9$ & $6,0, \overline{14}$ & $<5.8$ & -0.9 & $10,0, \overline{12}$ & $12 \cdot 3$ & $-10 \cdot 1$ \\
\hline $3,0,10$ & $<6.4$ & 0.5 & $6,0, \overline{16}$ & $11 \cdot 4$ & $12 \cdot 7$ & $10,0, \overline{14}$ & $<6.4$ & $2 \cdot 6$ \\
\hline
\end{tabular}

Table 3. Observed and calculated structure amplitudes for $0 \mathrm{kl}$ zone

\begin{tabular}{rrr}
$0 k l$ & \multicolumn{1}{c}{$F_{o}$} & $F_{c}$ \\
002 & $\mathbf{5 3 \cdot 4}$ & $\mathbf{6 4 \cdot 0}$ \\
004 & $\mathbf{5 4 \cdot 8}$ & $-53 \cdot 8$ \\
006 & $10 \cdot 4$ & $5 \cdot 0$ \\
008 & $18 \cdot 4$ & $-\mathbf{1 6 \cdot 5}$ \\
$0,0,10$ & $<6 \cdot 9$ & $-2 \cdot 3$ \\
$0,0,12$ & $19 \cdot 3$ & $15 \cdot 8$ \\
011 & $8 \cdot 1$ & $11 \cdot 9$ \\
012 & $38 \cdot 2$ & $53 \cdot 1$ \\
013 & $54 \cdot 7$ & $56 \cdot 0$ \\
014 & $36 \cdot 2$ & $35 \cdot 1$ \\
015 & $<4 \cdot 7$ & $-1 \cdot 9$ \\
016 & $6 \cdot 7$ & $-3 \cdot 2$ \\
017 & $<5 \cdot 7$ & $-5 \cdot 5$ \\
018 & $15 \cdot 3$ & $-9 \cdot 8$
\end{tabular}

\begin{tabular}{rrr}
$0 k l$ & \multicolumn{1}{c}{$F_{o}$} & \multicolumn{1}{c}{$\boldsymbol{F}_{c}$} \\
019 & 19.8 & 17.5 \\
$0,1,10$ & $<7.1$ & -6.6 \\
020 & 5.1 & 2.5 \\
021 & $<4.9$ & 3.9 \\
022 & 13.9 & 17.6 \\
023 & $<5.3$ & 6.7 \\
024 & 18.4 & 13.3 \\
025 & 16.9 & -11.0 \\
026 & $<6.1$ & -3.8 \\
027 & 8.7 & -14.4 \\
028 & 8.5 & -7.0 \\
029 & 9.7 & 8.0 \\
031 & 17.3 & 12.4 \\
032 & $<6.5$ & 4.2
\end{tabular}

\begin{tabular}{|c|c|c|}
\hline $0 k l$ & $F_{o}$ & $F_{c}$ \\
\hline 033 & $<6.7$ & $-4 \cdot 2$ \\
\hline 034 & $<6.9$ & $-2 \cdot 1$ \\
\hline 035 & $<7 \cdot 1$ & $5 \cdot 3$ \\
\hline 036 & $<7.3$ & $7 \cdot 2$ \\
\hline 037 & $<7.6$ & -5.8 \\
\hline 038 & $<7.9$ & 0.7 \\
\hline 039 & $10 \cdot 0$ & $-11 \cdot 7$ \\
\hline 040 & $<8.0$ & $-3 \cdot 2$ \\
\hline 041 & $<8.0$ & $-3 \cdot 1$ \\
\hline 042 & $<8.0$ & $6 \cdot 2$ \\
\hline 043 & $<8.1$ & $-2 \cdot 8$ \\
\hline 044 & $<8.1$ & $5 \cdot 2$ \\
\hline 045 & $<8.3$ & $5 \cdot 0$ \\
\hline & $12 \cdot 7$ & $-10 \cdot 6$ \\
\hline
\end{tabular}

chemistry, and approximately confirmed by the elec- resolved, and the atomic positions indicated must be tron-density map of Fig. $1(b)$. No individual atoms are regarded as tentative. 


\section{Discussion of the structure}

Atomic positions consistent with the electron-density maps of both projections are given in Table 1, and in Tables 2 and 3 are compared the observed and calculated structure amplitudes for the $h 0 l$ and $0 k l$ zones. The agreement residual $R=\Sigma|| F_{o}|-| F_{c}|| \div \Sigma\left|F_{o}\right|$ is 0.18 for the $h 0 l$ zone, and 0.20 for the $0 k l$ zone. These figures do not consider accidentally absent reflexions (two for $h 0 l$, one for $0 k l$ ) which according to the calculations should have been just visible.

The minimum distance of approach between neighbouring molecules varies from $3 \cdot 4 \AA$ to $3 \cdot 8 \AA$.

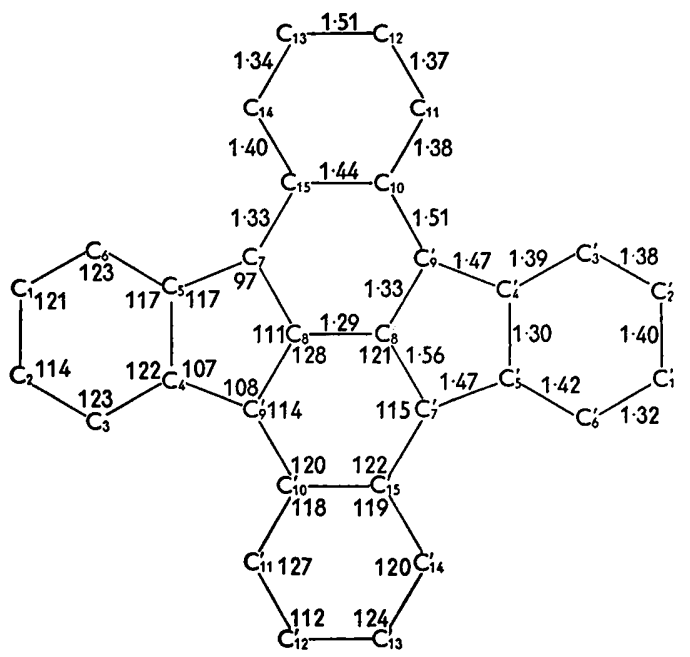

Fig. 2. Diagrammatic representation of the molecule, showing bond lengths (in Angström units) and angles (in degrees).

The atomic positions quoted are consistent with a molecule in which both the naphthacene and the phenyl groups are effectively planar. The plane of one makes an angle of about $8^{\circ}$ with that of the other, and the line of intersection contains the atoms $\mathrm{C}_{4}-\mathrm{C}_{5}$.

A diagrammatic representation of the molecule is given in Fig. 2; the average $\mathrm{C}-\mathrm{C}$ bond length is $1.40 \AA$, and individual bond lengths range from $1.29 \AA$ to $1.56 \AA$. The variation gives some indication of the probable accuracy of the atomic positions, and suggests that the statement concerning the departure of the molecule from planarity should be treated with some reserve.

The accuracy of the atomic positions is limited by the quality of the X-ray data, and by the poor resolution in the (100) projection. Some of the individual atoms might be resolved in the (101) projection, but three-dimensional methods would probably be necessary to determine the atomic positions accurately.

The authors wish to acknowledge their indebtedness to the following: Prof. Dufraise, of Paris, for providing a sample of diphenylene naphthacene; Dr I. G. Edmunds, of the Physics Department, College of Technology, for allowing access to some relevant data; Dr H. Lipson and Dr C. A. Taylor, of the same department, for valued advice and encouragement. One of us (A. W. H.) gratefully acknowledges an Imperial Chemical Industries Fellowship.

\section{References}

Hanson, A. W. (1953). Acta Cryst. 6, 35.

Hanson, A. W., Lipson, H. \& Taylor, C. A. (1953). Proc. Roy. Soc. A, 218, 371.

Hanson, A. W., Taylor, C. A. \& Lipson, H. (1952). Nature, Lond. 169, 1086.

\section{Short Communications}

Contributions intended for publication under this heading should be expressly so marked; they should not exceed about 500 words; they should be forwarded in the usual way to the appropriate Co-editor; they will be published as speedily as possible; and proofs will not generally be submitted to authors. Publication will be quicker if the contributions are without illustrations.

Acta Cryst. (1953). 6, 739

The crystal structure of $\boldsymbol{n}$-dodecylammonium chloride and bromide. By MANFRED GoRDon, Royal Technical College, Glasgow, Scotland, Einar Stenhagen, Medicinsk-Kemiska Institutionen, The University, Uppsala, Sweden and Vladimir Vand,* Chemistry Department, The University, Glasgow, Scotland

(Received 28 April 1953)

$N$-mono- $n$-alkyl substituted ammonium halides are of interest because of their monolayer behaviour and polymorphism, which suggests different kinds of packing of

* Imperial Chemical Industries Research Fellow. long hydrocarbon chains, and because the lower members possess interesting tetragonal high-temperature forms which are thought to exhibit some kind of chain rotation.

The shorter members of the series were studied first by Hendricks and others and more recently by King \& 